\title{
A comparison of pyogenic liver abscess in patients with or without diabetes: a retrospective study of 246 cases
}

\author{
Wenfei $\mathrm{Li}^{\dagger}$, Hongjie Chen ${ }^{\dagger}$, Shuai Wu and Jie Peng ${ }^{*}$ [D
}

\begin{abstract}
Background: Pyogenic liver abscess(PLA) has become common in patients with diabetes mellitus (DM), but it is unclear whether differences exist between patients with and without DM. A retrospective study was performed to identify these differences, summarize the clinical experience, and improve the diagnosis and treatment of PLA.

Methods: The patients were enrolled in a teaching hospital from January 2012 to December 2016. The patients were separated into two groups based on comorbidity with diabetes mellitus (DM). The DM group was further separated into two subgroups according to the $\mathrm{HbA1C}$ concentration to investigate whether glycaemic control affected the clinical characteristics of PLA patients with DM. Chi-square, Fisher's exact test, and t-tests were used to analyse and evaluate differences between the two groups.

Results: Two hundred and forty-six PLA patients were identified and 90 (36.6\%) had comorbid DM. Patients with DM were older, had higher levels of alkaline phosphatase and $\gamma$-glutamyl transferase, hypertension, a loss of body weight, a single abscess, and combined antibiotic therapy with the use of carbapenems and Klebsiella pneumoniae in their blood cultures but a less frequent history of abdominal surgery and Escherichia coli in their pus cultures. When DM patients were compared to non-DM patients, each of these differences was significant $(P<0.05)$. Diabetic PLA patients with poor glycaemic control had a significantly higher proportion of fever and both lobes $\operatorname{abscess}(P<0.05)$.

Conclusion: PLA patients with diabetes are older, have more serious complications, a higher prevalence of cardiovascular disease, an increased use of combined antibiotic therapy with carbapenem, and K. pneumoniae as the predominant pathogen, but these patients had fewer abdominal surgeries and fewer E. coli infections. In addition, poorly controlled glycaemia in diabetic PLA patients is associated with high incidence of fever and both lobes abscess.
\end{abstract}

Keywords: Pyogenic liver abscess, Diabetes, Diagnosis, Treatment

\section{Background}

Pyogenic liver abscess (PLA), which is a suppurating infection of the hepatic parenchyma, remains a condition associated with mortality and is reported in China and throughout the world, especially in Asia. The incidence rate of PLA is different worldwide and continues to increase annually [1]. In Taiwan, the annual all-age incidence of PLA has gradually increased from 10.83 to

\footnotetext{
*Correspondence: pjie138@163.com

${ }^{+}$Wenfei Li and Hongjie Chen contributed equally to this work.

Department of Infectious Diseases, Nanfang Hospital of Southern Medical

University, Guangzhou 510515, China
}

15.45 cases per 100,000 individuals from 2000 to 2011 [2]. In northeast China, an incidence of 5.7 cases per 100,000 individuals was reported in a large populationbased retrospective study [3]. In the United States, a large study described an incidence of 3.59 cases per 100,000 individuals [1]. PLA is concomitant with many diseases. These diseases are important risk factors and include diabetes mellitus (DM), malignancy, cholangitis, urinary tract disease, pneumonia, cardiovascular disease, autoimmune disease and malnutrition $[1,4-6]$. In recent years, PLA patients with concomitant DM have become more common in this hospital, and previous case reports

(c) The Author(s). 2018 Open Access This article is distributed under the terms of the Creative Commons Attribution 4.0 International License (http://creativecommons.org/licenses/by/4.0/), which permits unrestricted use, distribution, and 
demonstrate that DM results in an increased risk of PLA [7]. Tian et al. provided a comprehensive perspective of PLA [8], but whether differences exist among PLA patients with and without concomitant DM is unknown, especially in South China. Furthermore, there is little information regarding the effects of glycaemic control on the characteristics of PLA in diabetic patients. In addition, it is unknown whether glycaemic control affects the clinical characteristics of PLA with DM. Therefore, the purpose of this investigation was to compare the clinical characteristics of PLA patients with and without concomitant DM, to investigate whether glycaemic control affects the clinical characteristics of PLA patients with DM and to improve the diagnosis and treatment of PLA.

\section{Methods}

\section{Study population}

All of the hospitalized patients diagnosed with PLA (International Classification of Disease, Clinical Modification 572.0) and treated at the Nanfang Hospital of Southern Medical University in Guangzhou, China, from January 2012 to December 2016 were enrolled. This hospital is a public-care, teaching-medical centre in Guangzhou that serves as a patient referral centre and accepts patient referrals from every part of Guangzhou. The diagnosis of PLA was based on the following criteria: 1) clinical features, such as fever, chills, abdominal fullness, and abdominal pain; 2)etiological tests of the blood and the abscess; and 3)imaging evidence of the abscess cavity in the liver as judged by abdominal ultrasonography (US), computerized tomography (CT), or magnetic resonance imaging (MRI). Patients were excluded who did not have clear records or did not complete the treatment. The included patients were divided into two groups: those with and those without DM. The criteria for type II DM were defined according to the 2017 standards [9]: 1)fasting plasma glucose (FPG) $\geq 126 \mathrm{mg} / \mathrm{dL}(7.0 \mathrm{mmol} / \mathrm{L}) ; 2) 2$-h plasma glucose $(2-\mathrm{hPG}) \geq 200 \mathrm{mg} / \mathrm{dL}(11.1 \mathrm{mmol} / \mathrm{L}) ; 3) \mathrm{HbA} 1 \mathrm{C} \geq 6.5 \%$ (48 $\mathrm{mmol} / \mathrm{mol})$; and 4) a random plasma glucose $\geq 200 \mathrm{mg} / \mathrm{dL}(11.1 \mathrm{mmol} / \mathrm{L})$ in patients with classic symptoms of hyperglycaemia or hyperglycaemic crisis. To monitor glycaemic control, haemoglobin A1c (HbA1c) provides an estimate of average blood glucose during the preceding 3 months and is widely accepted as the primary indicator of the level of glycaemic control for the optimal management of diabetes [10]. The DM group was further categorised into two subgroups according to the $\mathrm{HbA} 1 \mathrm{C}$ concentration: group $1, \mathrm{HbA} 1 \mathrm{C}$ $<7.0 \%$, which indicated good glycaemic control, and group $2, \mathrm{HbA} 1 \mathrm{C} \geq 7 \%$, which indicated poor glycaemic control. The HbA1C cut-off value selection was based on previous studies [11].

\section{Data collection}

Data were collected by reviewing the medical records of each patient. The records included demographic characteristics (age and sex), length of stay, hospital stay, duration aetiology, underlying diseases, clinical parameters (signs and symptoms), HbA1C levels, laboratory values (hematologic, biochemical, and microbiological findings), imaging features, diagnoses, antimicrobial therapy, catheter drainage, and outcomes at discharge (i.e., recovered or died).

\section{Statistical analysis}

The statistical analysis was completed using the SPSS version 17.0 statistical software package(SPSS Inc., Chicago,Illinois, USA). All of the categorical variables were reported as percentages. The chi-square or Fisher's exact test was applied to evaluate the differences in the categorical variables. Continuous data were presented as the mean with the standard deviation (SD) and the Student's t-test was used to evaluate the differences in continuous variables. The statistical tests were performed with a two-tailed significance level of 0.05 .

\section{Results \\ Demographic characteristics}

A total of 286 patients received hospital treatment for PLA during the study period. A total of 12 patients were excluded who did not fit the inclusion criteria, 18 were excluded whose medical treatment data were incomplete, and 10 were excluded who were transferred to another hospital before the completion of the treatment. Ultimately, 246 patients were included in this retrospective study. The demographic characteristics and clinical features of the PLA patients are shown in Table 1. We found that males were predominant $(n=160,65 \%)$ and age ranged from 3 to 89 years with a mean age of $54.2 \pm$ 14.2 years. The length of the hospital stay was 3 to 71 days with an average of $18.5 \pm 11.4$ days. Of the 246 patients, $90(36.6 \%)$ had DM and 64 of these were men with a male-to-female ratio of $2.5: 1.0$. The mean age was $56.5 \pm 10.9$ years (26-84 years) for the DM group, which was higher than the $52.9 \pm 15.6$ years (3-89 years)for the non-DM group $(P=0.039)$. However, there was no difference in gender between the two groups $(P=0.129)$. Table 5 shows the clinical features in diabetic patients with good controlled or poorly controlled glycaemia. All of the diabetic patients had recorded $\mathrm{HbA1C}$ levels. Based on the HbA1C levels of the diabetic patients, 27 patients (30.0\%) had good glycaemic control and 67 (70.0\%) had poor glycaemic control. In our study, the age and gender were not identified between the controlled glycaemia groups (Table 5). 
Table 1 Characteristics and clinical findings for patients with pyogenic liver abscess with diabetes mellitus (DM) or without DM (non-DM)

\begin{tabular}{|c|c|c|c|}
\hline Characteristic & DM cases (\%) & Non-DM cases (\%) & $P$ value (chi-square test) \\
\hline \multicolumn{4}{|l|}{ Sex } \\
\hline Male & $64(71.1)$ & $96(61.5)$ & 0.129 \\
\hline Female & $26(28.9)$ & $60(38.5)$ & \\
\hline Age (year) (mean $\pm S D)$ & $56.5 \pm 10.9$ & $52.9 \pm 15.6$ & $0.039^{a}$ \\
\hline \multicolumn{4}{|l|}{ Underlying conditions } \\
\hline Gallbladder diseases & $42(53.3)$ & $81(51.9)$ & 0.427 \\
\hline Hypertension & $24(26.7)$ & $17(10.9)$ & 0.001 \\
\hline Gastrointestinal surgery & $3(3.3)$ & $20(12.8)$ & 0.025 \\
\hline Liver surgery & $1(1.1)$ & $14(9.0)$ & 0.013 \\
\hline Pulmonary tuberculosis & $3(3.3)$ & $4(2.6)$ & 1.000 \\
\hline \multicolumn{4}{|l|}{ Symptoms } \\
\hline Fever & $80(88.9)$ & 138(88.5) & 0.919 \\
\hline Chills & $58(64.4)$ & 93(59.6) & 0.454 \\
\hline Abdominal pain & $48(53.3)$ & $90(57.7)$ & 0.507 \\
\hline Frailty & $33(36.7)$ & $57(36.5)$ & 0.984 \\
\hline Nausea or Vomiting & 10(11.1) & $23(14.7)$ & 0.421 \\
\hline Cough & 13(14.4) & 17(10.9) & 0.413 \\
\hline Weight loss & $18(20.0)$ & $13(8.3)$ & 0.008 \\
\hline
\end{tabular}

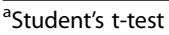

\section{Underlying diseases}

Although most patients in both groups had gallbladder diseases $(n=123,50.0 \%)$ (i.e., gallstones, choledocholithiasis, chronic cholecystitis, pancreatitis, or postcholecystectomy), no significant differences were found between the groups. Hypertension ( $n=41,16.7 \%$ ) was the second most common underlying disease and was more common in the DM group ( $26.7 \%$ vs. $10.9 \%, P=0.001)$. This was followed by gastrointestinal surgery $(n=23,9.3 \%)$, liver surgery $(n=15$, $6.1 \%)$, pulmonary tuberculosis $(n=7,2.5 \%)$, nephrotic syndrome $(\mathrm{n}=1,0.4 \%)$ and hyperthyroidism $(\mathrm{n}=1,0.4 \%)$. Patients with DM had a lower prevalence of gastrointestinal surgery (3.3\% vs. $12.8 \%, P=0.025)$ and liver surgery $(1.1 \%$ vs. $9.0 \%, P=0.027$ ) (Table 1 ). However, the underlying disease did not differ between the good controlled and poorly controlled glycaemia groups (Table 5).

\section{Clinical features}

In both groups, the most common symptom was fever ( $n=218,88.6 \%$ ) with $88.9 \%$ in the DM group and $88.5 \%$ in the non-DM group; this symptom was followed by chills ( $n=151,61.4 \%)$, abdominal pain $(n=138,56.1 \%)$, frailty ( $n=90,36.6 \%)$, nausea or vomiting ( $n=33,13.4 \%)$, weight loss $(n=31,12.6 \%)$, cough $(n=30,12.2 \%)$, abdominal fullness $(n=8,3.3 \%)$ and jaundice $(n=5,2.0 \%)$ in decreasing order. The DM group had a higher prevalence of body weight loss $(20.0 \%$ vs. $8.3 \%, P=0.008)$ (Table 1$)$, but there were no significant differences among the glycaemic control groups. However, patients with poorly controlled glycaemia had a higher rate of fever $(77.8 \%$ vs. 93.7\%, $P=0.028$ ) (Table 5).

\section{Laboratory examination}

In both groups, inflammatory biomarkers were generally elevated and included C-reactive protein (CRP), procalcitonin (PCT), erythrocyte sedimentation rate (ESR), and leukocyte and neutrophil count. Remarkably, the sensitivity of the erythrocyte sedimentation rate was $100 \%$ positive in all of the PLA patients. In addition, $\mathrm{C}$-reactive protein and procalcitonin were approximately $100 \%$ positive in all PLA patients. A higher incidence of elevated alanine aminotransferase (ALT), aspartate aminotransferase (AST), total bilirubin (T.Bil), alkaline phosphatase (ALP), $\gamma$-glutamyl transferase (GGT) and decreased albumin (ALB)levels were detected in both groups. Additionally, patients with DM had a higher prevalence of ALP $(73.5 \%$ vs. $49.2 \%, P=0.020)$ and GGT(91.2\% vs. $46.2 \%, P=0.001$ ) (Table 2). However, no laboratory examination was identified in the group with controlled glycaemia (Table 5).

\section{Imaging}

All of the cases were imaged by ultrasonography, computerized tomography $(\mathrm{CT})$, or magnetic resonance imaging (MRI), but no significant difference was found between the groups. According to these images, most of the lesions ( $n=171,69.5 \%)$ were located in the right lobe $(70.0 \%$ in the DM group and $69.2 \%$ in the non-DM 
Table 2 Laboratory and image findings for pyogenic liver abscess patients with diabetes mellitus (DM) or without DM (non-DM)

\begin{tabular}{|c|c|c|c|}
\hline Characteristic & DM cases (\%) & Non-DM cases (\%) & $P$ value (chi-square test) \\
\hline \multicolumn{4}{|l|}{ Laboratory findings } \\
\hline WBC > $9.5(\times 10 \wedge 9 / L)$ & 64/90(71.1) & 96/156(61.5) & 0.129 \\
\hline WBC < $3.5(\times 10 \wedge 9 / L)$ & 1/90(1.1) & 4/156(2.6) & $0.397^{\mathrm{a}}$ \\
\hline NEUT> 75\% & 62/90(68.9) & $101 / 156(64.7)$ & 0.508 \\
\hline Anaemiab $^{b}$ & 71/90(78.9) & 113/156(72.4) & 0.262 \\
\hline $\mathrm{PLT}>350(\times 10 \wedge 9 / \mathrm{L})$ & 28/90(31.1) & 59/156(37.8) & 0.289 \\
\hline $\mathrm{PLT}<125(\times 10 \wedge 9 / \mathrm{L})$ & 12/90(13.3) & 23/156(14.7) & 0.760 \\
\hline$\uparrow E S R(m m / 1 h)$ & $27 / 27(100)$ & $32 / 32(100)$ & 1.000 \\
\hline $\mathrm{CRP}>5(\mathrm{mg} / \mathrm{L})$ & 81/83(97.6) & 131/137(95.6) & 0.449 \\
\hline $\mathrm{PCT}>0.05(\mathrm{ng} / \mathrm{ml})$ & $66 / 66(100)$ & 90/92(97.8) & $0.510^{\mathrm{a}}$ \\
\hline T.Bil > 20.5 $(\mu \mathrm{mol} / \mathrm{L})$ & 29/89(32.6) & 39/155(25.2) & 0.468 \\
\hline ALT> 50(U/L) & 32/90(35.6) & 54/155(34.8) & 0.910 \\
\hline $\mathrm{AST}>40(\mathrm{U} / \mathrm{L})$ & 27/90(30.0) & 57/154(37.0) & 0.266 \\
\hline $\mathrm{ALP}>125(\mu \mathrm{mol} / \mathrm{L})$ & 25/34(73.5) & $32 / 65(49.2)$ & 0.020 \\
\hline $\mathrm{GGT}>60(\mathrm{U} / \mathrm{L})$ & 31/34(91.2) & 30/65(46.2) & 0.001 \\
\hline $\mathrm{ALB}<40(\mathrm{~g} / \mathrm{L})$ & 87/90(96.7) & 142/155(91.6) & 0.123 \\
\hline \multicolumn{4}{|l|}{ Abscess location } \\
\hline Right lobe & 63/90(70.0) & 108/156(69.2) & 0.232 \\
\hline Left lobe & 16/90(17.8) & 29/156(18.6) & \\
\hline Both lobes & 9/90(10.0) & 19/156(12.2) & \\
\hline Caudate lobe & $2 / 90(2.2)$ & $0(0)$ & \\
\hline \multicolumn{4}{|l|}{ Abscess size $(\mathrm{cm})$} \\
\hline$<5$ & 21/90(23.3) & 37/156(23.7) & 0.907 \\
\hline $5-10$ & 49/90(54.5) & 88/156(56.4) & \\
\hline$>10$ & 20/90(22.2) & 31/156(19.9) & \\
\hline \multicolumn{4}{|l|}{ Count of abscess } \\
\hline Single & 75/90(83.3) & 110/156(70.5) & 0.025 \\
\hline Multiple & 15/90(16.7) & 46/156(29.5) & \\
\hline
\end{tabular}

WBC white blood cell count, NEUT neutrophil count, $P L T$ platelets, ESR erythrocyte sedimentation rate, $P C T$ procalcitonin, $C R P C$-reactive protein, $A L T$ alanine aminotransferase, AST aspartate aminotransferase, T.Bil total bilirubin, ALP alkaline phosphatase, GGT $\gamma$-glutamyl transferase, ALB albumin

${ }^{a}$ Fisher's exact test; ${ }^{b}$ Haemoglobin $<130 \mathrm{~g} / \mathrm{l}$ in men, $<115 \mathrm{~g} / \mathrm{l}$ in women; $\uparrow E S R>15 \mathrm{~mm} / 1 \mathrm{~h}$ in men, $>20 \mathrm{~mm} / 1 \mathrm{~h}$ in women

group) and the diameters ranged from $0.8 \times 0.6 \mathrm{~cm}$ to $19.0 \times 17.7 \mathrm{~cm}$. Patients with a single abscess $(n=185$, $75.2 \%$ ) were three-fold as common as patients with multiple abscesses $(n=61,24.8 \%)$, and the DM group had a higher prevalence of a single abscess $(83.3 \%$ vs. $70.5 \%$, $P=0.025)$; however, the difference was not statistically significant for either abscess lesion number or size (Table 2). Remarkably, patients with poor glycaemic control had a higher prevalence in both lobes and the left lobe but less in the right lobe abscess $(14.3 \%$ vs. $0,23.8 \%$ vs. $3.7 \%$, and $60.3 \%$ vs. $92.6 \%$, respectively, $P=0.010$ ) (Table 5).

\section{Aetiology}

Blood cultures were collected from 118 patients and $24.6 \%$ (29 cases) were positive. The DM group had a higher positive proportion(36\% vs. $16.2 \%, P=0.013)$. Only $6.9 \%$ (two cases) of the culture-positive patients had polymicrobial growth. Pus cultures were collected from 121 patients and the overall positive growth rate was $58.7 \%$ (71 cases). Only 4.2\%(three cases)had polymicrobial growth. A total of 9 cases were positive for both blood and pus cultures. A total of 5 cases had monomicrobial infections and 4 cases had polymicrobial infections. Among the culture-positive patients, 105 strains were identified, which included 10 Gram-positive organisms (9.5\%) and 95 Gram-negative organisms (90.5\%). Klebsiella pneumoniae was the most common pathogen identified in both blood and pus cultures for both groups and had a higher prevalence in blood cultures $(26 \%$ vs. $1.5 \%, P=0.001)$. The second most common pathogen was Escherichia coli, which was less 
frequently isolated from pus cultures of the DM group $(16 \%$ vs. $2.2 \%, P=0.037)$. Other pathogens were isolated from $<5 \%$ of the patients (Table 3 ). However, $K$. pneumoniae infections and $E$. coli infections showed no significant differences between the glycaemic control groups in our study (Table 5).

\section{Treatments and outcomes}

Antibiotic therapy was the most common treatment for both groups $(n=241,98.0 \%)$, which included 46 patients (19.1\%) who received a single antibiotic and 195 patients (80.9\%) who received a combination antibiotic therapy. The remaining five patients only accepted simple abscess drainage. In both groups, the most frequently used antibiotics were third generation cephalosporins (including ceftriaxone, ceftazidime, or cefoperazone) ( $n=167,69.3 \%)$, which were followed by fluoroquinolone (including levofloxacin, moxifloxacin, or ciprofloxacin) ( $n=72,29.9 \%)$, carbapenems (imipenem or meropenem) $(n=64,26.6 \%)$, or combined with metronidazole $(n=109,45.2 \%)$. It is notable that compared to the non-DM group, the DM group had a significantly higher frequency of combined antibiotic therapy $(86.7 \%$ vs. $75.0 \%, P=0.009)$ with carbapenems $(36.7 \%$ vs. $19.9 \%, P=0.004)$. In addition, percutaneous drainage was performed in $134(54.5 \%)$ patients and surgical drainage was performed in 22 patients (8.9\%), but there were no differences between the groups. The total effective rate of the therapy was $96.3 \%(237 / 246)$ and the two groups had similar rates. A total of 6 cases were invalid and 3 cases died from septic shock or multiple organ failure (MOF). No significant difference was noted in the ratio of the effective treatment and the mortality between the groups (Table 4). Furthermore, the treatment strategy, hospitalization days and mortality were not significantly different between the controlled glycaemia groups either (Table 5).

\section{Discussion}

The morbidity of patients with PLA and diabetes has recently increased. This may be due to reduced immunity, neutrophil chemotaxis, mononuclear phagocyte activation, and/or opsonization in diabetes patients. In addition, hyperglycaemia can promote bacterial growth in tissues, and metabolic disorders impact the liver, gut, pancreas, stomach, and intestine, which induces biliary disease. DM is a risk factor for PLA with a hazard risk rate of 3.6 to 9 -fold $[6,7]$ and it is relatively common in PLA patients with reported co-existence rates of $30 \%$ in Hong Kong [12], 31\% in Canada [13], 28.7\% in a single centre in Xi'an, China [14], 23\% in Italy [15], and 36.6\% in this study.

The majority of the patients in this study were males with a mean of $54.2 \pm 14.2$ years old, which is comparable with other studies [16]. Patients with DM were approximately 4 years older than patients without DM, which was similar to previous reports [17] and may relate to the fact that most DM patients are older and immunocompromised. Notably, biliary tract diseases were the major underlying disease process, which indicated that biliary infections were the predominate cause of

Table 3 Microbiological isolates in blood and pus cultures from patients with diabetes mellitus (DM) or without DM (non-DM)

\begin{tabular}{|c|c|c|c|c|c|c|c|}
\hline \multirow[t]{2}{*}{ Characteristic } & \multicolumn{3}{|l|}{ Blood culture } & \multicolumn{3}{|l|}{ Pus culture } & \multirow[t]{2}{*}{ Strains } \\
\hline & $\mathrm{DM}(n=50)$ & Non-DM $(n=68)$ & $P$ value (chi-square) & $\mathrm{DM}(n=46)$ & Non-DM $(n=75)$ & $P$ value (chi-square) & \\
\hline Positive growth & 18 & 11 & 0.013 & 28 & 43 & 0.701 & - \\
\hline Polymicrobial growth & 1 & 1 & 1.000 & 1 & 2 & 1.000 & - \\
\hline Gram-positive aerobes & & & & & & & $10(9.5)$ \\
\hline Staphylococcus & 2 & 0 & $0.177^{a}$ & 1 & 2 & 1.000 & $5(4.7)$ \\
\hline Streptococcus & 1 & 0 & $0.424^{a}$ & 0 & 2 & $0.525^{\mathrm{a}}$ & $3(2.9)$ \\
\hline Enterococcus & 0 & 2 & $0.507^{\mathrm{a}}$ & 0 & 0 & - & $2(1.9)$ \\
\hline Gram-negative organisms & & & & & & & $95(90.5)$ \\
\hline Klebsiella pneumonia & 13 & 1 & 0.001 & 23 & 25 & 0.069 & $62(59.0)$ \\
\hline Escherichia coli & 2 & 7 & 0.357 & 1 & 12 & 0.037 & $22(21.0)$ \\
\hline Pseudomonas & 0 & 1 & $1.000^{\mathrm{a}}$ & 0 & 1 & $1.000^{\mathrm{a}}$ & $2(1.9)$ \\
\hline Aeroenterobacter & 0 & 0 & - & 2 & 1 & 0.665 & $3(2.9)$ \\
\hline Burkholderia cepacia & 1 & 0 & $0.424^{\mathrm{a}}$ & 1 & 0 & $0.380^{\mathrm{a}}$ & $2(1.9)$ \\
\hline Acinetobacter baumannii & 0 & 0 & - & 1 & 0 & $0.380^{\mathrm{a}}$ & $1(0.95)$ \\
\hline Enterobacter cloacae & 0 & 0 & - & 0 & 1 & $1.000^{\mathrm{a}}$ & $1(0.95)$ \\
\hline Bacillus citrate & 0 & 1 & $1.000^{\mathrm{a}}$ & 0 & 0 & - & $1(0.95)$ \\
\hline Shewanella putrefaciens & 0 & 0 & - & 0 & 1 & $1.000^{\mathrm{a}}$ & $1(0.95)$ \\
\hline
\end{tabular}


Table 4 Treatment and outcome in pyogenic liver abscess patients with diabetes mellitus (DM) or without DM (non-DM)

\begin{tabular}{|c|c|c|c|}
\hline Characteristic & DM cases $(\%)$ & Non-DM cases (\%) & $P$ value (chi-square test) \\
\hline \multicolumn{4}{|l|}{ Antibiotic option } \\
\hline Combined & $78(86.7)$ & $117(75.0)$ & 0.009 \\
\hline Single & $9(0.1)$ & $37(23.7)$ & \\
\hline \multicolumn{4}{|l|}{ Antibiotic drugs } \\
\hline The third generation of cephalosporin & $63(70.0)$ & 104(66.7) & 0.590 \\
\hline Fluoroquinolone & $33(36.7)$ & $39(25.0)$ & 0.053 \\
\hline Carbapenems & $33(36.7)$ & $31(19.9)$ & 0.004 \\
\hline Metronidazole & $43(47.8)$ & $66(42.3)$ & 0.405 \\
\hline \multicolumn{4}{|l|}{ Method of abscess drainage } \\
\hline Percutaneous drainage & $51(56.7)$ & $83(53.2)$ & 0.599 \\
\hline Surgical drainage & $7(7.8)$ & 15(9.6) & 0.627 \\
\hline \multicolumn{4}{|l|}{ Clinical outcomes } \\
\hline Cured & 85(94.4) & 152(97.4) & 0.395 \\
\hline Death & $1(1.1)$ & $2(1.3)$ & 1 \\
\hline
\end{tabular}

PLA, and this is consistent with a previous study from east China [18]. In addition, patients with DM had a higher prevalence of hypertension, which suggests that PLA patients with DM were more likely to have cardiovascular disease. In this investigation, gastrointestinal operations included appendectomy $(n=10)$, enterectomy $(n=7)$, laparotomy $(n=4)$ and hemigastrectomy $(n=2)$, and were more common in the non-DM group, which indicated that the gastrointestinal operation history for PLA patients without diabetes was relevant, especially for appendectomy [19]. In our study, liver surgery included a partial hepatectomy $(n=11)$, transcatheter arterial chemoembolization (TACE) $(n=3)$ and splenectomy $(n=1)$. Although no significant difference was found between the groups, TACE and splenectomy have been linked with certain PLA, which were statistically significant independent risk factors [20, 21]. In addition, patients without DM have been reported to have a higher prevalence of biliary tract diseases [17], but this investigation failed to support that finding.

In our study, the main clinical findings for PLA patients were fever, chills, and abdominal pain, which is consistent with other studies [13, 16-18, 22]. Clinical features, such as cough, jaundice, frailty, and abdominal fullness are not typical and may relate to a delayed diagnosis of PLA. The loss of body weight was higher in the DM group and this may be because DM patients ineffectively use glucose, which results in increased consumption of body fat and protein, and this metabolic condition may enhance more infection. In addition, we found that poorly controlled glycaemia patients were prone to have a fever, which is different from previous studies [23] indicating that glycaemia control affects the severity of PLA.
The laboratory outcomes did not differ between the groups. Most patients had elevated white blood cell counts (WBC), neutrophil counts, C-reactive protein (CRP), procalcitonin (PCT), erythrocyte sedimentation rate (ESR) levels, and abnormal liver function tests, but there was no significant difference between the groups. The CRP, PCT and ESR levels appeared to be more sensitive than WBC. Collectively, the analysis of these biomarkers may reduce the misdiagnosis of PLA. Furthermore, the alkaline phosphatase and $\gamma$-glutamyl transferase levels of the DM group were higher, which suggests that liver injury in PLA patients with DM was more remarkable with more fatty liver and biliary cell damage. Nevertheless, our study showed that no laboratory examination was significantly identified between good and poorly controlled glycaemia.

We all know that imaging is crucial for the diagnosis of PLA. In this investigation, most liver abscess was singular and located in the right lobe with a diameter of $5-10 \mathrm{~cm}$, which was in agreement with previous studies $[14,17]$. This may be due to the large area of the right liver lobe and its propensity to receive the most portal blood flow [17]. Interestingly, unlike the non-DM group, the overwhelming majority of DM patients had a single abscess. This may be because patients with non-DM had a higher incidence of previous surgeries with possible abdominal infection involving other areas of the liver. It is also interesting to note that patients with poorly controlled glycaemia had a higher rate of both lobes. This may be because poorly controlled glycaemia can help bacteria grow, which makes the overall condition worse. Thus, it is important to control good glycaemia.

Gram-negative bacteria predominated in this investigation, which indicates that antibiotics against Gram-negative 
Table 5 Baseline characteristics, clinical presentation, and outcome of diabetic patients with good or poorly controlled glycaemia

\begin{tabular}{|c|c|c|c|}
\hline Characteristic & Good control of glycaemia $(n=27)(\%)$ & Poor control of glycaemia $(n=63)(\%)$ & $P$ value (chi-square test) \\
\hline Male & $20(74.1)$ & $44(69.4)$ & 0.685 \\
\hline Age (year) $($ mean $\pm S D)$ & $57.2 \pm 2.0$ & $56.1 \pm 1.4$ & 0.681 \\
\hline \multicolumn{4}{|l|}{ Underlying conditions } \\
\hline Gallbladder diseases & $15(55.6)$ & $27(42.9)$ & 0.268 \\
\hline Hypertension & $7(25.9)$ & $17(26.9)$ & 0.917 \\
\hline Abdominal surgery & $2(7.4)$ & $1(1.6)$ & 0.159 \\
\hline Liver surgery & $0(0)$ & $1(1.6)$ & 0.510 \\
\hline Pulmonary tuberculosis & $1(3.7)$ & $2(3.2)$ & 0.909 \\
\hline \multicolumn{4}{|l|}{ Symptoms } \\
\hline Fever & $21(77.8)$ & $59(93.7)$ & 0.028 \\
\hline Chills & $14(51.9)$ & $44(69.8)$ & 0.102 \\
\hline Abdominal pain & $14(51.9)$ & $34(54.0)$ & 0.854 \\
\hline Weight loss & $5(18.5)$ & $13(20.6)$ & 0.818 \\
\hline \multicolumn{4}{|l|}{ Laboratory findings } \\
\hline WBC > $9.5(\times 10 \wedge 9 / L)$ & 18/27 (66.7) & 46/63 (73.0) & 0.543 \\
\hline NEUT> 75\% & $16 / 27(59.3)$ & 46/63 (73.0) & 0.196 \\
\hline Anaemia $^{\mathrm{b}}$ & 22/27 (81.5) & 49/63 (77.8) & 0.693 \\
\hline $\mathrm{PLT}>350(\times 10 \wedge 9 / \mathrm{L})$ & $3 / 27(11.1)$ & 9/63 (14.3) & 0.946 \\
\hline $\mathrm{PLT}<125(\times 10 \wedge 9 / \mathrm{L})$ & $11 / 27(40.7)$ & 17/63 (27.0) & 0.196 \\
\hline$\uparrow E S R(m m / 1 ~ h)$ & $7 / 7(100)$ & 20/20 (100) & 1.000 \\
\hline CRP > 5(mg/L) & 25/25 (100) & 56/58 (96.6) & $1.000^{\mathrm{a}}$ \\
\hline $\mathrm{PCT}>0.05(\mathrm{ng} / \mathrm{ml})$ & 20/20 (100) & 46/46 (100) & 1.000 \\
\hline T.Bil > 20.5 $(\mu \mathrm{mol} / \mathrm{L})$ & $11 / 27(40.7)$ & 18/62 (29.0) & 0.279 \\
\hline $\mathrm{ALT}>50(\mathrm{U} / \mathrm{L})$ & $11 / 27(40.7)$ & 21/63 (33.3) & 0.501 \\
\hline $\mathrm{AST}>$ 40(U/L) & 9/27 (33.3) & 18/63 (28.6) & 0.919 \\
\hline $\mathrm{ALP}>125(\mu \mathrm{mol} / \mathrm{L})$ & $6 / 10(60)$ & 19/24 (79.2) & 0.467 \\
\hline $\mathrm{GGT}>60(\mathrm{U} / \mathrm{L})$ & 9/10 (90) & 22/24 (91.7) & $1.000^{\mathrm{a}}$ \\
\hline $\mathrm{ALB}<40(\mathrm{~g} / \mathrm{L})$ & 26/27 (96.3) & 61/63 (96.8) & $1.000^{\mathrm{a}}$ \\
\hline \multicolumn{4}{|l|}{ Abscess locations } \\
\hline Right lobe & $25(92.6 \%)$ & 38 (60.3\%) & 0.010 \\
\hline Left lobe & $1(3.7 \%)$ & $15(23.8 \%)$ & \\
\hline Both lobes & $0(0)$ & $9(14.3 \%)$ & \\
\hline Caudate lobe & $1(3.7 \%)$ & $1(1.6 \%)$ & \\
\hline \multicolumn{4}{|l|}{ Abscess size $(\mathrm{cm})$} \\
\hline$<5$ & $4(14.8)$ & $16(25.4)$ & 0.526 \\
\hline $5-10$ & $16(59.3)$ & $34(54.0)$ & \\
\hline$>10$ & $7(25.9)$ & $13(20.6)$ & \\
\hline Multiple abscesses & $4(14.8)$ & $11(17.5)$ & 0.758 \\
\hline K. Pneumonia infections & 6/18 (33.3) & $25 / 50(50)$ & 0.223 \\
\hline E.coli infections & 1/18 (5.6) & 2/50 (4) & $1.000^{\mathrm{a}}$ \\
\hline
\end{tabular}


Table 5 Baseline characteristics, clinical presentation, and outcome of diabetic patients with good or poorly controlled glycaemia (Continued)

\begin{tabular}{llll}
\hline Characteristic & Good control of glycaemia $(n=27)(\%)$ & Poor control of glycaemia $(n=63)(\%)$ & $P$ value (chi-square test) \\
\hline Treatment & & & 0.889 \\
Percutaneous drainage & $15(55.6)$ & $36(57.1)$ & 0.932 \\
Surgical drainage & $2(7.4)$ & $5(7.9)$ & 0.848 \\
Antibiotics only & $10(37.0)$ & $22(35.0)$ & 0.132 \\
Clinical outcomes & & $58(92.1)$ & $1.000^{\mathrm{a}}$ \\
Cured & $27(100)$ & $1(1.6)$ & 0.252 \\
Death & $0(0)$ & $19.4 \pm 1.3$ & \\
Hospitalization days & $22.3 \pm 2.3$ & & \\
\hline
\end{tabular}

WBC white blood cell count, NEUT neutrophil count, PLT platelets, ESR erythrocyte sedimentation rate, $P C T$ procalcitonin, CRP C-reactive protein, $A L T$ alanine aminotransferase, AST aspartate aminotransferase, T.Bil total bilirubin, ALP alkaline phosphatase, GGT $\gamma$-glutamyl transferase, ALB albumin

${ }^{a}$ Fisher's exact test; ${ }^{b}$ Haemoglobin $<130 \mathrm{~g} / \mathrm{l}$ in men, $<115 \mathrm{~g} / \mathrm{l}$ in women; $\uparrow E S R>15 \mathrm{~mm} / 1 \mathrm{~h}$ in men, $>20 \mathrm{~mm} / 1 \mathrm{~h}$ in women

bacteria should be used for empiric therapy. In addition, we also found that K. pneumoniae was the dominant pathogen (accounting for $59.0 \%$ of the pathogens), which is consistent with previous studies from Asian countries where it accounted for $40-80 \%$ [16, 24-26]. Followed by E. coli,Staphylococcus, E. aerogenes, and Streptococcus. In this investigation, the prevalence of $K$. pneumoniae in blood cultures was higher in the DM group. These findings are consistent with previous studies and could be explained by possible intimal vascular defects in DM patients [17]. In contrast, PLA patients without DM had a higher prevalence of $E$. coli in pus cultures, which is similar to a previous study by Tian et al. [8], which may be correlated with a higher incidence of post-abdominal operation and resulting liver infection. Remarkably, the incidence of positive pus cultures was similar between the two groups. Positive pus cultures were significantly higher than blood cultures, which is similar to a previous report $[8,14]$. These findings imply that bacteria were primarily confined to the liver. The administration of antibiotics and anti-fever drugs was significantly lower than the incidence of positive blood cultures. Positive blood cultures were higher in the DM group, which suggests that PLA patients with DM are more likely to develop blood infections or even sepsis. Previous studies have shown that diabetic PLA patients with poor glycaemic control had higher K. pneumoniae infection rates [23], but our study failed to support this finding. In our study, $98.0 \%$ of the patients underwent antibiotic therapy, 54.5\% underwent percutaneous drainage, and $8.9 \%$ underwent surgical drainage, which resulted in an effective rate of $96.7 \%$ and a fatality rate of $1.2 \%$, which was similar to recent international reports of $0.9-2.5 \%[5,16,18]$. For treatment, third generation cephalosporin or fluoroquinolone combined with metronidazole was used. Carbapenems were used if the outcomes were not optimal or if patients were in a critical condition. Remarkably, 5 patients presented with severe sepsis and 4 patients required an ICU stay and they all received combination antibiotic therapy including carbapenems for broad coverage. It is notable that the DM group had a higher proportion of antibiotic combined therapy and carbapenems and a higher likelihood of severe complications with difficult to control infections. These observations suggest that PLA patients with DM may need more aggressive combined therapy with carbapenems. However, there was not a significant difference between percutaneous drainage and surgical therapy for the two groups. Our study showed that the treatment strategy, hospitalization days and mortality were not significantly different between the controlled glycaemia groups. Based on this investigation, antibiotic therapy and catheter drainage are the appropriate treatments for PLA patients with or without DM.

There were notable limitations to this study. This was a retrospective study, it was performed in a single centre, and the results may not be generalizable. However, the results are based on a large number of cases and should be valuable to other investigators and clinicians.

\section{Conclusions}

In conclusion, PLA was mainly due to biliary tract disease with a single lesion located in the right lobe, and the predominant pathogen was $K$. pneumonia. PLA patients with and without DM had many differing clinical characteristics. PLA patients with DM were older and had more complications including a higher prevalence of cardiovascular disease, a loss of body weight, K. pneumonia infections, antibiotic combined therapy with carbapenem, and a greater likelihood of sepsis. In contrast, a history of gastrointestinal surgery and E. coli were less frequent. Furthermore, diabetic PLA patients with poor glycaemic control had a significantly higher proportion of fever and both lobes abscess. Additional large-scale studies and fundamental research can build upon this investigation and should provide further insight into PLA. 


\section{Abbreviations}

2-hPG: 2-h plasma glucose; ALB: Albumin; ALP: Alkaline phosphatase; ALT: Alanine aminotransferase; AST: Aspartate aminotransferase; CRP: CReactive protein; CT: computerized tomography; DM: Diabetes mellitus; $E$. coli: Escherichia coli; ESR: Erythrocyte sedimentation rate; FPG: Fasting plasma glucose; GGT: $\gamma$-Glutamyl transferase; MRI: Magnetic resonance imaging: NEUT: Neutrophil count; PCT: Procalcitonin; PLA: Pyogenic liver abscess; PLT: Platelets; SD: Standard deviation; T.Bil: Total bilirubin: US: Ultrasonography; WBC: White blood cell count

\section{Availability of data and materials}

The datasets used and/or analysed during the current study are available from the corresponding author upon reasonable request.

\section{Authors' contributions}

Study conception and design: JP and WFL. Acquisition, analysis and/or interpretation of data: WFL and HJC. Drafting/revision of the work for intellectual content and context: WFL, HJC and SW. Final approval and overall responsibility for the published work: JP. All of the authors read and approved the final manuscript.

\section{Ethics approval and consent to participate}

The study was approved by the clinical research ethics committee of Nanfang Hospital of Southern Medical University. Written informed consent was obtained by all of the participants.

\section{Consent for publication}

Not applicable.

\section{Competing interests}

The authors declare that they have no competing interests.

\section{Publisher's Note}

Springer Nature remains neutral with regard to jurisdictional claims in published maps and institutional affiliations.

Received: 17 May 2018 Accepted: 25 September 2018

Published online: 01 October 2018

\section{References}

1. Meddings L, Myers RP, Hubbard J, Shaheen AA, Laupland KB, Dixon E, Coffin C, Kaplan GG. A population-based study of pyogenic liver abscesses in the United States: incidence, mortality, and temporal trends. Am J Gastroenterol. 2010;105(1):117-24.

2. Chen Y, Lin C, Chang S, Shi Z. Epidemiology and clinical outcome of pyogenic liver abscess: an analysis from the National Health Insurance Research Database of Taiwan, 2000-2011. J Microbiol Immunol Infect. 2016; 49(5):646-53.

3. Finch RG, Blasi FB, Verheij TJ, Goossens H, Coenen S, Loens K, Rohde G, Saenz $\mathrm{H}$, Akova M. GRACE and the development of an education and training curriculum. Clin Microbiol Infect. 2012;18(9):E308-13.

4. Gallagher MC, Andrews MM. Postdischarge outcomes of pyogenic liver abscesses: single-center experience 2007-2012. Open Forum Infect Dis. 2017; 4(3):x159.

5. Rahimian J, Wilson T, Oram V, Holzman RS. Pyogenic liver abscess: recent trends in etiology and mortality. Clin Infect Dis. 2004;39(11):1654-9.

6. Tsai FC, Huang YT, Chang LY, Wang JT. Pyogenic liver abscess as endemic disease, Taiwan. Emerg Infect Dis. 2008;14(10):1592-600.

7. Thomsen RW, Jepsen P, Sorensen HT. Diabetes mellitus and pyogenic liver abscess: risk and prognosis. Clin Infect Dis. 2007;44(9):1194-201.

8. Tian LT, Yao K, Zhang XY, Zhang ZD, Liang YJ, Yin DL, Lee L, Jiang HC, Liu LX. Liver abscesses in adult patients with and without diabetes mellitus: an analysis of the clinical characteristics, features of the causative pathogens, outcomes and predictors of fatality: a report based on a large population, retrospective study in China. Clin Microbiol Infect. 2012;18(9):E314-30.

9. American Diabetes Association. 2. Classification and Diagnosis of Diabetes: Standards of Medical in Diabetes 2018. Diabetes Care. 2018;41(Suppl 1):S13-27.

10. American Diabetes Association. Standards of medical care in diabetes--2014 Diabetes Care. 2014;37(Suppl 1):S114-80.
11. Lin YT, Wang FD, Wu PF, Fung CP. Klebsiella pneumoniae liver abscess in diabetic patients: association of glycemic control with the clinical characteristics. BMC Infect Dis. 2013;13:56.

12. Yu SC, Ho SS, Lau WY, Yeung DT, Yuen EH, Lee PS, Metreweli C. Treatment of pyogenic liver abscess: prospective randomized comparison of catheter drainage and needle aspiration. HEPATOLOGY. 2004;39(4):932-8.

13. Kaplan GG, Gregson DB, Laupland KB. Population-based study of the epidemiology of and the risk factors for pyogenic liver abscess. Clin Gastroenterol Hepatol. 2004;2(11):1032-8.

14. Du ZQ, Zhang LN, Lu Q, Ren YF, LV Y, Liu XM, Zhang XF. Clinical Charateristics and outcome of pyogenic liver abscess with different size: 15-year experience from a single center. Sci Rep. 2016;6:35890.

15. Serraino C, Elia C, Bracco C, Rinaldi G, Pomero F, Silvestri A, Melchio R, Fenoglio LM. Characteristics and management of pyogenic liver abscess: a European experience. Medicine (Baltimore). 2018;97(19):e628.

16. Kong H, Yu F, Zhang W, Li X. Clinical and microbiological characteristics of pyogenic liver abscess in a tertiary hospital in East China. Medicine (Baltimore). 2017;96(37):e8050.

17. Foo NP, Chen KT, Lin HJ, Guo HR. Characteristics of pyogenic liver abscess patients with and without diabetes mellitus. Am J Gastroenterol. 2010; 105(2):328-35

18. Liu L, Chen W, Lu X, Zhang K, Zhu C. Pyogenic liver abscess: a retrospective study of 105 cases in an emergency department from East China. J EMERG MED. 2017:52(4):409-16

19. Liao KF, Lai SW, Lin CL, Chien SH. Appendectomy correlates with increased risk of pyogenic liver abscess: a population-based cohort study in Taiwan. Medicine (Baltimore). 2016;95(26):e4015.

20. Lai SW, Lai HC, Lin CL, Liao KF. Splenectomy correlates with increased risk of pyogenic liver abscess: a Nationwide cohort study in Taiwan. J EPIDEMIOL. 2015;25(9):561-6.

21. Oshima S, Tani N, Takaishi K, Hirano M, Makari Y, Hoshi M, Doi T, Matsuno H, Kobori Y. Kobayashi T et al: [clinical evaluation of the risk factors for liver abscess after TACE or RFA]. Gan To Kagaku Ryoho. 2014:41(12):2113-5.

22. Ali AH, Smalligan RD, Ahmed M, Khasawneh FA. Pyogenic liver abscess and the emergence of Klebsiella as an etiology: a retrospective study. Int J Gen Med. 2013;7:37-42.

23. Liao WI, Sheu WH, Chang WC, Hsu CW, Chen YL, Tsai SH. An elevated gap between admission and A1C-derived average glucose levels is associated with adverse outcomes in diabetic patients with pyogenic liver abscess. PLoS One. 2013;8(5):e64476.

24. Lok KH, Li KF, Li KK, Szeto ML. Pyogenic liver abscess: clinical profile, microbiological characteristics, and management in a Hong Kong hospital. Jicrobiol Immunol Infect. 2008;41(6):483-90

25. Cerwenka H. Pyogenic liver abscess: differences in etiology and treatment in Southeast Asia and Central Europe. World J Gastroenterol. 2010;16(20):2458-62.

26. Luo M, Yang XX, Tan B, Zhou XP, Xia HM, Xue J, Xu X, Qing Y, Li CR, Qiu JF, et al. Distribution of common pathogens in patients with pyogenic liver abscess in China: a meta-analysis. Eur J Clin Microbiol Infect Dis. 2016;35(10): 1557-65.

Ready to submit your research? Choose BMC and benefit from

- fast, convenient online submission

- thorough peer review by experienced researchers in your field

- rapid publication on acceptance

- support for research data, including large and complex data types

- gold Open Access which fosters wider collaboration and increased citations

- maximum visibility for your research: over $100 \mathrm{M}$ website views per year

At BMC, research is always in progress.

Learn more biomedcentral.com/submission 